\title{
Intraoperative infusion of branched-chain amino acids in patients undergoing gastrointestinal tumor surgery
}

\author{
Qiwei $\mathrm{Wu}^{1,2}$, Yanying Zhang ${ }^{1,2}$, Yan Yang ${ }^{1,2}$, Shengjin $\mathrm{Ge}^{1,2^{*}}$ and Zhanggang Xue
}

\begin{abstract}
Background: The purpose of this study is to investigate the effects of intraoperative infusion of branched-chain amino acids (BCAA) in patients undergoing gastrointestinal tumor surgery.

Methods: Sixty-one patients with gastrointestinal tumors undergoing gastrointestinal surgery were enrolled and randomly assigned to receive an intraoperative infusion of 3-compound BCAA solution ( $N=20)$, amino acids (AA) solution ( $N=21)$, or normal saline (NS) $(N=20)$. Nasopharyngeal temperature, blood glucose $(B G)$, plasma insulin, and blood free fatty acids (FFA) concentrations were measured at 30 min before and 10 min after induction $\left(T_{0,}, T_{1}\right)$, 30 min and $2 \mathrm{~h}$ after skin incision $\left(T_{2}, T_{3}\right)$, and $1 \mathrm{~h}$ after tracheal extubation $\left(T_{4}\right)$. Intensity of shivering and pain was accessed at $1 \mathrm{~h}$ after extubation.

Results: The temperature in the BCAA and AA group was significantly higher than that in the NS group at $T_{4}$ $(P=0.014$ and 0.033$)$. The incidence of shivering in the BCAA and AA group was significantly lower than in the NS group ( $P=0.027$ and 0.012 ). BG increased in AA group at $T_{3}$ and $T_{4}(P=0.001$ and 0.045$)$. The plasma insulin concentration increased in the BCAA and AA group from $T_{1}$ to $T_{3}$. The plasma FFA concentrations in the BCAA group were lower than in the AA and NS group from $T_{2}$ to $T_{4}$.
\end{abstract}

Conclusions: Intraoperative BCAA and AA infusion alleviated postoperative hypothermia and shivering. BCAA infusion also inhibited fat mobilization, without adversely affecting blood glucose.

Trial registration: ChiCTR-TRC-14004668

Keywords: Metabolism-glucose, Metabolism-lipid, Temperature-metabolism

\section{Background}

During anesthesia and surgery, redistribution hypothermia occurs as peripheral vessels dilate, and impaired hypothalamic thermoregulation further worsens the hypothermia $[1,2]$. Thereafter, core body temperature gradually declines as heat is lost from the surface of the body, and the production of heat slows [3-6]. Furthermore, the stress of surgery and anesthesia leads to a series of changes at the metabolic level and in the neuro-endocrine axis $[2,7]$. Catabolism exceeds anabolism, thereby increasing the physiological burden of patients undergoing surgery.

\footnotetext{
* Correspondence: ge.shengjin@fudan.edu.cn

${ }^{1}$ Department of Anaesthesia, Zhongshan Hospital, Fudan University, No. 180 Fenglin Road, Shanghai 200032, China

${ }^{2}$ Department of Anaesthesiology, Shanghai Medical College, Fudan

University, Shanghai 200032, China
}

Unlike other amino acids (AAs) that are metabolized dominantly in the liver, the branched-chain amino acids (BCAA), isoleucine, leucine, and valine are uniquely metabolized primarily in the skeletal muscle. Moreover, BCAAs can promote anabolism without placing additional burdens on the liver. In previous studies, infusions of compound intravenous AA solutions have been shown to exhibit a thermogenic effect during anesthesia [8-10]. Therefore, we hypothesized that intraoperative infusion of BCAA instead of AAs could provide even more benefit to patients. The objective of our study was to investigate the effects of intraoperative infusion of BCAA on body temperature, carbohydrate metabolism, and lipid 
metabolism in patients undergoing gastrointestinal (GI) tumor surgery.

\section{Methods \\ Patients}

The study was approved by the Ethics Committee of Zhongshan Hospital, Fudan University (B2014-013) and registered at www.chictr.org.cn (ChiCTR-TRC-14004668). Written informed consent was obtained from all patients. We screened a total of 69 consecutive patients (aged 1865 years and American Society of Anesthesiologist physical status classes I-II) scheduled to undergo GI surgery (stomach or colorectal) at Zhongshan Hospital from May to July 2014. Patients were excluded if they had a serum albumin $<35 \mathrm{~g} \cdot \mathrm{L}^{-1}$, anemia (hemoglobin $<100 \mathrm{~g} \cdot \mathrm{L}^{-1}$ ), or evidence of a major medical condition (such as diabetes mellitus, thyroid disease, hepatic disease, or renal disease). They were also excluded if they were receiving drugs known to have metabolic effects, such as corticosteroids or $\beta$-blockers. Only 61 patients fulfilled all criteria. They were randomly assigned at a 1:1:1 ratio to one of three groups using random number method: BCAA infusion group (compound amino acid injection, BBCA Pharmaceutical Co. Ltd., Anhui, China; see Appendix), AA infusion group (Aminoplasmal, B. Braun Melsungen AG, Jiangsu, China; see in Appendix), and normal saline (NS) infusion group (normal saline, Baxter Healthcare Co. Ltd., Shanghai, China). All patients underwent standard GI preoperative procedures, including overnight fasting. Patients scheduled for colorectal operations also underwent bowel-cleansing procedures the day before surgery. No premedications were given except intestinal antibacterial drugs, which were started 3 days preoperatively.

\section{Anesthesia}

Before the induction of general anesthesia, each patient received an epidural catheter between $T_{8}$ and $T_{12}$, a central venous catheter, and a radial artery catheter. Hemodynamic monitoring was performed using a three-lead electrocardiogram monitor, finger pulse oximetry, and continuous arterial pressure measurement via the radial artery catheter.

General anesthesia was induced in all patients with fentanyl $3 \mu \mathrm{g} \cdot \mathrm{kg}^{-1}$, propofol $2 \mathrm{mg} \cdot \mathrm{kg}^{-1}$, and lidocaine $60 \mathrm{mg}$, and rocuronium $0.6 \mathrm{mg} \cdot \mathrm{kg}^{-1}$ was administered to facilitate intubation. After tracheal intubation, mechanical ventilation was initiated with $50 \%$ oxygen: $50 \%$ air-gas mixture, and ventilation was adjusted to maintain the end-tidal $\mathrm{CO}_{2}$ at $30-$ $40 \mathrm{mmHg}$. After induction, $6-10 \mathrm{~mL}$ of $0.5 \%$ bupivacaine was administered in increments through the epidural catheter, and epidural anesthesia was maintained with intermittent boluses of 3-5 mL every hour during the operation. General anesthesia was maintained with sevoflurane at a minimum alveolar concentration of 0.8-0.9. Boluses of phenylephrine or ephedrine were administered if necessary to maintain the heart rate and arterial pressure within $\pm 30 \%$ of baseline. Each patient received $0.125 \%$ bupivacaine and fentanyl $2 \mu \mathrm{g} \cdot \mathrm{mL}^{-1}$ for patient-controlled epidural analgesia postoperatively.

\section{Experimental protocol}

The operating room temperature was maintained at $21-23{ }^{\circ} \mathrm{C}$. No other perioperative warming strategies were used except the blood products that were warmed to $42{ }^{\circ} \mathrm{C}$ if a transfusion was needed. A forced-air warming system would be used as a rescue method, if the body core temperature dropped below $35{ }^{\circ} \mathrm{C}$. All patients were covered with two layers of quilt postoperatively.

The AA infusion was diluted to $4.26 \%$ with normal saline. The total dose of BCAA was calculated as $5.6 \mathrm{~mL} \cdot \mathrm{kg}^{-1} \cdot \mathrm{h}^{-1}$ according to the basal metabolic rate, $4 \mathrm{~kJ} \cdot \mathrm{kg}^{-1} \cdot \mathrm{h}^{-1}$. The intervention fluid (BCAA, $\mathrm{AA}$, or NS) was infused at a rate of $5.6 \mathrm{~mL} \cdot \mathrm{kg}^{-1} \cdot \mathrm{h}^{-1}$. In addition, Lactated Ringer's solution (lot no. S1401082; Baxter Healthcare Co. Ltd., Shanghai, China) was administered at $4 \mathrm{~mL} \cdot \mathrm{kg}^{-1} \cdot \mathrm{h}^{-1}$ as the basal fluid infusion. All intervention fluids were infused via a central venous catheter, using a Graseby 3500 pump (SIMS Graseby Ltd., Watford, UK). The infusions were initiated at the beginning of induction and discontinued when the patient was extubated.

The BCAA, AA, and NS solutions were prepared by a specified investigator. The other investigators, all health-care providers, and all patients were blinded to the preparation procedure and were thereby unaware of the group assignments.

\section{Measurements}

The core temperature was measured continuously using a nasopharyngeal temperature probe (DatexOhmeda Division, Instrumentarium Corp., Helsinki, Finland) which was inserted from the nostril with the distance from the philtrum to the tragus. Blood samples were collected $30 \mathrm{~min}$ before induction $\left(T_{0}\right)$, $10 \mathrm{~min}$ after induction $\left(T_{1}\right), 30 \mathrm{~min}$ and $2 \mathrm{~h}$ after skin incision $\left(T_{2}\right.$ and $\left.T_{3}\right)$, and $1 \mathrm{~h}$ after extubation $\left(T_{4}\right)$. Each blood sample was transferred immediately to an EDTA tube and centrifuged at $4{ }^{\circ} \mathrm{C}$ until analyzed within $24 \mathrm{~h}$ by an automatic biochemical analyzer (7080, Hitachi, Japan) and automatic electrochemiluminescence analyzer (Cobas e602, Roche, Swiss). At the same time points, the blood glucose concentration was measured by a glucose analyzer (One Touch Sure-step; LifeScan, 
Johnson \& Johnson Medical Co. Ltd., Shanghai, China) using a sample of blood obtained from the fingertips. At $1 \mathrm{~h}$ after tracheal extubation, the intensity of shivering was assessed by a single investigator (who was the same for each patient) using the Wrench grade for post-anesthetic shivering [11] (Additional file 1: Table S1), and the severity of pain was assessed using a visual analog scale (with $0=$ no pain and $10=$ worst pain imaginable) [12].

\section{Sample size estimation and statistical analysis}

In this study, the primary outcome measure was nasopharyngeal temperature, and the secondary outcome measures were blood glucose concentration and circulating concentrations of plasma insulin and free fatty acids (FFA). PASS 11.0 (NCSS, Kaysville, Utah, USA) were used for sample size estimation and power calculation. In previous studies [10], the standard deviation of body temperature was approximately 0.1-0.2 ${ }^{\circ} \mathrm{C}$, and the expected difference between groups was approximately $0.3-0.5{ }^{\circ} \mathrm{C}$; therefore, less than nine patients in each group were calculated to be sufficient for this outcome (power $80 \%, \alpha=0.05$ ). On the basis of an expected difference in blood glucose concentration of $1 \mathrm{mmol} \cdot \mathrm{L}^{-1}$ between the two groups (standard deviation $=1 \mathrm{mmol} \cdot \mathrm{L}^{-1}$; power $80 \%$, $\alpha=0.05), 17$ patients in each group were calculated to be sufficient for this study $[10,13,14]$. Thus, 20 patients in each group were deemed sufficient for this study. Statistical analyses were performed using SPSS 20.0 (SPSS, Inc.) software. Categorical variables, including gender and type of operation, were analyzed using $\chi^{2}$ or Fisher's exact test. Continuous variables were analyzed using two-tailed general linear model repeated measures analysis of variance. Probability $(p)$ values $<0.05$ were considered statistically significant.

\section{Results}

There were no baseline differences among the three groups for gender, age, body mass index (BMI), type of operation, duration of surgery, and anesthesia (Table 1). The severity of pain $1 \mathrm{~h}$ after surgery between the two groups did not reach statistical significance. No patient received a blood transfusion in this study. The mean postoperative hospitalization days were $7.9 \pm 1.8,8.1 \pm 1.9$, and $8.6 \pm 2.4$ days for the BCAA, AA, and NS groups, respectively. Only one patient in the AA group suffered from bowel leak in hospitalization within 30 days after operation. The continuous data were normally distributed, with homogeneity of variance (data not shown). The baseline data (30 min before anesthesia) for core temperature, blood glucose, plasma insulin, and FFA level were not significantly different among the three groups (Table 2).

The patients' core temperature in both the BCAA and NS groups slowly decreased during surgery while none dropped below $35{ }^{\circ} \mathrm{C}$. However, the mean temperature in the BCAA and AA groups was significantly higher at $1 \mathrm{~h}$ after extubation than in the NS group $(36.39,36.34$ vs $36.05, P=0.029$, power $=80.76 \%$ ) (Table 2). There was no significant difference in incidence and intensity of shivering (grade 0 vs grade $\geq 1$ ) between the BCAA and $\mathrm{AA}$ group. BCAA and $\mathrm{AA}$ groups were significantly

Table 1 Baseline characteristics of the three groups

\begin{tabular}{|c|c|c|c|c|}
\hline & BCAA $(N=20)$ & $\mathrm{AA}(N=21)$ & NS $(N=20)$ & $P$ \\
\hline Gender & & & & 0.509 \\
\hline Male & $13(65.0)$ & $10(47.6)$ & $12(60.0)$ & \\
\hline Female & $7(35.0)$ & $11(52.4)$ & $8(40.0)$ & \\
\hline Age (years) & 56.5 (18 to 65$)$ & 58 (40 to 68$)$ & 52 (34 to 65) & 0.313 \\
\hline $\mathrm{BMI}\left(\mathrm{kg} / \mathrm{m}^{2}\right)$ & $23.62 \pm 2.33$ & $23.32 \pm 3.02$ & $22.83 \pm 2.33$ & 0.594 \\
\hline Surgery type ${ }^{a}$ & & & & 0.710 \\
\hline Gastric & $8(40.0)$ & $10(47.6)$ & $7(35.0)$ & \\
\hline Colorectal & $12(60.0)$ & $11(52.4)$ & $13(65.0)$ & \\
\hline Duration of surgery (min) & $146.75 \pm 2.92$ & $147.14 \pm 22.89$ & $137.37 \pm 36.41$ & 0.428 \\
\hline Total volume of fluid $(\mathrm{mL})$ & $2615.00 \pm 241.21$ & $2504.76 \pm 423.64$ & $2457.90 \pm 287.36$ & 0.006 \\
\hline \multicolumn{5}{|l|}{ Amino acids } \\
\hline Non-BCAA (g) & 0 & $26.35 \pm 5.55$ & 0 & \\
\hline BCAA (g) & $38.62 \pm 7.75$ & $11.29 \pm 2.38$ & 0 & \\
\hline
\end{tabular}

Data are mean \pm standard deviation or number (percentage)

Abbreviations: $B C A A$ branched-chain amino acids, $B M I$ body mass index, NS normal saline, AA amino acid

${ }^{\mathrm{a} S u r g e r i e s}$ were conducted open 
Table 2 Changes in nasopharyngeal temperature and plasma concentrations of glucose, insulin, and free fatty acids

\begin{tabular}{|c|c|c|c|c|c|}
\hline Factors & Variables & $\mathrm{BCAA}(N=20)$ & $\mathrm{AA}(N=21)$ & NS $(N=20)$ & $p^{*}$ \\
\hline \multirow[t]{5}{*}{$T\left({ }^{\circ} \mathrm{C}\right)$} & $T_{0}$ & $36.77 \pm 0.37$ & $36.56 \pm 0.35$ & $36.61 \pm 0.37$ & 0.177 \\
\hline & $T_{1}$ & $36.51 \pm 0.31$ & $36.40 \pm 0.35$ & $36.35 \pm 0.39$ & 0.335 \\
\hline & $T_{2}$ & $36.21 \pm 0.35$ & $36.26 \pm 0.35$ & $36.16 \pm 0.47$ & 0.709 \\
\hline & $T_{3}$ & $35.89 \pm 0.42$ & $36.17 \pm 0.36$ & $35.82 \pm 0.56$ & 0.040 \\
\hline & $T_{4}$ & $36.39 \pm 0.40$ & $36.34 \pm 0.34$ & $36.05 \pm 0.49$ & 0.029 \\
\hline \multirow[t]{5}{*}{$\mathrm{BG}\left(\mathrm{mmol} \cdot \mathrm{L}^{-1}\right)$} & $T_{0}$ & $5.12 \pm 0.89$ & $5.11 \pm 0.45$ & $4.83 \pm 0.92$ & 0.436 \\
\hline & $T_{1}$ & $5.47 \pm 0.78$ & $5.48 \pm 0.56$ & $5.15 \pm 1.02$ & 0.349 \\
\hline & $T_{2}$ & $5.87 \pm 0.92$ & $6.32 \pm 0.61$ & $5.92 \pm 1.39$ & 0.297 \\
\hline & $T_{3}$ & $5.96 \pm 0.95$ & $7.61 \pm 1.24$ & $6.05 \pm 2.07$ & 0.001 \\
\hline & $T_{4}$ & $6.09 \pm 1.33$ & $7.21 \pm 1.28$ & $7.05 \pm 1.84$ & 0.045 \\
\hline \multirow[t]{5}{*}{ Insulin $\left(\mu \mathrm{U} \cdot \mathrm{mL}^{-1}\right)$} & $T_{0}$ & $4.38 \pm 2.40$ & $4.41 \pm 3.69$ & $3.34 \pm 2.50$ & 0.276 \\
\hline & $T_{1}$ & $6.85 \pm 4.46$ & $6.87 \pm 5.46$ & $3.17 \pm 3.03$ & 0.016 \\
\hline & $T_{2}$ & $6.86 \pm 4.78$ & $16.12 \pm 12.25$ & $2.18 \pm 2.05$ & 0.000 \\
\hline & $T_{3}$ & $12.72 \pm 7.83$ & $28.89 \pm 21.10$ & $2.57 \pm 1.62$ & 0.000 \\
\hline & $T_{4}$ & $10.56 \pm 5.53$ & $8.55 \pm 6.09$ & $4.03 \pm 2.98$ & 0.001 \\
\hline \multirow[t]{5}{*}{ FFA $\left(m m o \mid \cdot L^{-1}\right)$} & $T_{0}$ & $0.86 \pm 0.30$ & $0.80 \pm 0.37$ & $0.96 \pm 0.29$ & 0.288 \\
\hline & $T_{1}$ & $0.66 \pm 0.26$ & $0.59 \pm 0.25$ & $0.78 \pm 0.26$ & 0.075 \\
\hline & $T_{2}$ & $0.47 \pm 0.14$ & $0.39 \pm 0.16$ & $0.59 \pm 0.23$ & 0.003 \\
\hline & $T_{3}$ & $0.42 \pm 0.19$ & $0.89 \pm 0.30$ & $0.74 \pm 0.37$ & 0.000 \\
\hline & $T_{4}$ & $0.37 \pm 0.19$ & $0.58 \pm 0.24$ & $0.86 \pm 0.40$ & 0.000 \\
\hline
\end{tabular}

Data are represented as mean \pm standard deviation. Italicized texts indicate significance

Abbreviations: $T$ nasopharyngeal temperature, $B G$ blood glucose, $F F A$ free fatty acids, $B C A A$ branched-chain amino acids, NS normal saline, $A A$ amino acids, $T_{0}$

$30 \mathrm{~min}$ before induction, $T_{1} 10 \mathrm{~min}$ after induction (before skin incision), $T_{2}$ and $T_{3} 30 \mathrm{~min}$ and $2 \mathrm{~h}$ after skin incision, $T_{4} 1 \mathrm{~h}$ after extubation

*P mean value among three groups (one way ANOVA)

different from NS group ( $P$ values were 0.027 and 0.012 , respectively Table 3 ).

Blood glucose concentrations increased after the induction of anesthesia in all three groups. There were no significant differences between the BCAA and NS groups over time, although the values in the BCAA group seemed to be more stable than those in the NS group. BG in the AA group peaked at $T_{3}$ (7.61 $\mathrm{mM})$ and were higher at $T_{3}$ and $T_{4}$ comparing

Table 3 Shivering grades at $1 \mathrm{~h}$ after extubation

\begin{tabular}{llllll}
\hline Group & Grade & & & & \\
\cline { 2 - 6 } & 0 & 1 & 2 & 3 & 4 \\
\hline BCAA $(N=20)$ & 14 & 5 & 1 & 0 & 0 \\
AA $(N=21)$ & 15 & 4 & 2 & 0 & 0 \\
NS $(N=20)$ & 7 & 4 & 5 & 3 & 1 \\
\hline
\end{tabular}

In comparison of shivering grades (grade 0 vs grade $\geq 1$ ), BCAA and AA groups were significantly different from the NS group $(P$ value were 0.027 and 0.012 , respectively.). There was no significant difference between $B C A A$ and $A A$ groups $(P=0.920)$

Abbreviations: $B C A A$ branched-chain amino acids, $A A$ amino acids, NS normal saline with the BCAA group $(P=0.001$ and 0.045 , power $=$ 99.96). The plasma insulin concentration of BCAA and AA groups began to increase at $T_{1}$ and peaked at $T_{3}$. The plasma insulin in the NS group stayed in baseline level during surgery. The insulin values were significantly higher in BCAA and AA patients than in NS patients from $T_{1}$ to $T_{4}$ (all $P<0.05$, power $=99.80$, Table 2). Moreover, in AA patients, the insulin level increased to $28.89 \mu \mathrm{U} / \mathrm{mL}$ at $T_{3}$.

The FFA concentrations in the NS and AA groups decreased at the beginning of anesthesia, hit a nadir at $30 \mathrm{~min}$ after skin incision, and then slowly increased until $1 \mathrm{~h}$ after extubation. By contrast, plasma FFA concentrations in the BCAA group decreased from $10 \mathrm{~min}$ after induction to the end of surgery and remained low thereafter (Table 2).

\section{Discussion}

Nutrients such as glucose, fatty acids, and AAs are not usually administered intraoperatively because of the stress response that typically accompanies anesthesia 
and surgery [15]. However, recent studies have demonstrated that administration of AAs can stimulate energy expenditure and enhance thermogenesis during surgery and anesthesia [8, 9, 16-19]. Furthermore, the optimal composition of intraoperative AAs remains poorly understood. Our current results suggest that BCAA administration can alleviate postoperative shivering. Moreover, BCAA infusion had little influence on the blood glucose concentration, thereby suggesting that it is safe during anesthesia and surgery from this perspective.

In our previous study of patients undergoing surgery under general anesthesia combined with epidural block, the thermogenic effects of intraoperative AAs infusion were not significant in the early part of surgery; they were most obvious at $2 \mathrm{~h}$ after the induction of anesthesia [14]. This was consistent with our results. The current study, which was the first randomized controlled trial to investigate the thermogenic effects of BCAA in patients, showed that BCAA infusion also had a delayed thermogenic response. Furthermore, the BCAA infusion was also noted to decrease the incidence of postoperative shivering as AA infusion did.

Previous studies in humans [20], as well as in an animal model [10], have reported substantial increases in blood glucose and insulin concentrations during the administration of AAs. This phenomenon was reproducible in our study. Although our current results demonstrated a statistically significant increase in blood glucose while infusing BCAA, the increase was not as dramatic as that in the AA group. Moreover, in the AA group, insulin level increased significantly as well. Previous studies demonstrated that plasma AA concentration was closely associated with insulin resistance [21-24]. Several experiments on human and rats suggested that the increased plasma AA concentration reduced insulin-induced peripheral glucose disposal by interfering with muscle glucose transport and phosphorylation [25, 26]. This phenomenon was observed in the present study. In the BCAA and AA groups, although plasma insulin level largely increased after BCAA or AA infusion, the plasma glucose concentration was still upregulated. In other words, glucose utilization decreased. In clinical practice, a blood glucose concentration of $6-10 \mathrm{mmol} \cdot \mathrm{L}^{-1}$ would be considered acceptable. Our study demonstrated that BCAA infusion had less effect on blood glucose than AA did.

As a consequence of the endocrine response to stress, lipid mobilization is enhanced [27]. In the current study, the FFA concentration in the BCAA group declined during surgery, whereas the FFA concentration in the NS group decreased at $T_{2}$ but subsequently rose at $T_{3}$. This may reflect a decreased lipid mobilization effect of BCAA. Although detailed mechanisms must still be uncovered, one explanation may be that lipolysis is suppressed by the enhanced insulin secretion trigged by BCAA [28]. In the AA group, FFA concentration was unstable during surgery. This might due to the influence of complicated component of AA on lipid mobilization.

Limitations of this study should be clarified. Although the sample size estimation showed that our number of subjects was adequate for statistical analysis, our sample size was small and validation is required in a larger population. Furthermore, we did not obtain a muscle biopsy to investigate the direct results of $\mathrm{BCAA}$ metabolism in the skeletal muscle, and further studies, including invasive approaches for studying BCAA metabolism, are still needed. Also, the long-term complications of patients who underwent GI surgery were not included in this study. It remains unknown whether the effect of BCAA on insulin induction could have detrimental effect on type 2 diabetes or even pre-diabetic patients.

\section{Conclusions}

In conclusion, our findings indicate that an intraoperative infusion of BCAA can alleviate hypothermia and the intensity of shivering after GI surgery. It can also inhibit fat mobilization, without adversely affecting blood glucose concentrations in our cohort. Further multicenter external validation is needed.

\section{Statement of ethics and consent}

The study was approved by the Ethics Committee of Zhongshan Hospital, Fudan University (B2014-013) and registered at www.chictr.org.cn (ChiCTR-TRC-14004668). Written informed consent was obtained from all patients to participate under the "Ethics, consent, and permissions" heading and to publish from the participant to report individual patient data.

\section{Appendix}

Table 4 Composition of the 3-compound branched-chain amino acids injection (per $1000 \mathrm{ml}$ )

\begin{tabular}{ll}
\hline Amino acids & Values $(\mathrm{g})$ \\
\hline L-isoleucine & 13.50 \\
L-leucine & 16.50 \\
L-valine & 12.60 \\
Total amino acids & 42.60 \\
\hline
\end{tabular}

Osmotic pressure: approximately $382 \mathrm{mOsm} / \mathrm{L}$ 
Table 5 Composition of the compound amino acids injection (Aminoplasmal, per $1000 \mathrm{ml}$ )

\begin{tabular}{ll}
\hline Amino acids & Values (g) \\
\hline L-Alanine & 8.30 \\
L-Valine & 10.60 \\
L-Leucine & 13.60 \\
L-Isoleucine & 8.80 \\
L-Phenylalanine & 1.60 \\
L-Tryptophane & 1.50 \\
L-Methionine & 1.20 \\
L-Proline & 7.10 \\
Glycine & 6.30 \\
L-Ornithine & 1.35 \\
L-Serine & 3.70 \\
L-Threonine & 4.60 \\
L-Cysteine & 0.59 \\
L-Tyrosine & 0.67 \\
L-Histidine & 4.70 \\
L-Arginine & 8.80 \\
L-Aspartic acid & 2.50 \\
L-Asparagine & 0.48 \\
L-Glutamic acid & 5.70 \\
L-lysine acetate & 10.60 \\
Total amino acids & 100 \\
\hline Osmotic &
\end{tabular}

Osmotic pressure: approximately $875 \mathrm{mOsm} / \mathrm{L}$

\section{Additional file}

Additional file 1: Table S1. It is a five-point scale for shivering grade assessment, published by Wrench IJ et al in Anaesthesia on 1997. (DOC $31 \mathrm{~kb})$

\section{Competing interests}

The authors declare no potential or real conflicts of interest.

\section{Authors' contributions}

QW: study design, data collection and analysis, and writing up of the first draft of the manuscript. $Y Z$ and $Y Y$ : patient recruitment and data collection. SG and ZX: participating in the manuscript drafting and revising. All authors read and approved the final manuscript.

\section{Acknowledgements}

This work is attributed to and financially supported by the Department of Anaesthesia, Zhongshan Hospital, Fudan University, Shanghai, China.

Received: 13 July 2015 Accepted: 9 December 2015

Published online: 15 December 2015

\section{References}

1. Reynolds L, Beckmann J, Kurz A. Perioperative complications of hypothermia. Best Pract Res Clin Anaesthesiol. 2008;22:645-57.

2. Borsook D, George E, Kussman B, Becerra L. Anesthesia and perioperative stress: consequences on neural networks and postoperative behaviors. Prog Neurobiol. 2010;92:601-12.
3. Matsukawa T, Sessler DI, Sessler AM, Schroeder M, Ozaki M, Kurz A, et al. Heat flow and distribution during induction of general anesthesia. Anesthesiology. 1995;82:662-73.

4. Sessler DI. Perioperative heat balance. Anesthesiology. 2000;92:578-96.

5. Kurz A, Sessler DI, Christensen R, Dechert M. Heat balance and distribution during the core-temperature plateau in anesthetized humans. Anesthesiology. 1995;83:491-9.

6. Matsukawa T, Sessler DI, Christensen R, Ozaki M, Schroeder M. Heat flow and distribution during epidural anesthesia. Anesthesiology. 1995;83:961-7.

7. Nygren J. The metabolic effects of fasting and surgery. Best Pract Res Clin Anaesthesiol. 2006:20:429-38.

8. Moriyama T, Tsuneyoshi I, Omae T, Takeyama M, Kanmura Y. The effect of amino-acid infusion during off-pump coronary arterial bypass surgery on thermogenic and hormonal regulation. J Anesth. 2008;22:354-60.

9. Kamitani K, Yoshida H, Takagi M, Minami M, Tokutake M, Kishi R, et al. [Effect of amino acid infusion for prevention of intraoperative hypothermia during laparotomy]. Masui. 2006;55:1216-21.

10. Jin L, Ge S, Wang H, Gao X, Jin J, Xue Z. Metabolic effects of intraoperative amino acid infusion in mongrel dogs. Ann Nutr Metab. 2012;61:117-25.

11. Wrench IJ, Singh P, Dennis AR, Mahajan RP, Crossley AW. The minimum effective doses of pethidine and doxapram in the treatment of post-anaesthetic shivering. Anaesthesia. 1997:52:32-6.

12. Dundee JW. Clinical evaluation of mild analgesics. $\mathrm{Br} J$ Clin Pharmacol. 1980;10 Suppl 2:3295-34S

13. Chow SC, Wang H, Shao J. Sample size calculations in clinical research. Taylor \& Francis, Britain; 2003.

14. Zhong J, Ge SJ, Zhuang XF, Cang J, Xue ZG. Effect of intraoperative amino acid infusion on blood glucose under general anesthesia combined with epidural block. Ann Nutr Metab. 2012;61:1-6.

15. Thorell A, Nygren J, Ljungqvist O. Insulin resistance: a marker of surgical stress. Curr Opin Clin Nutr Metab Care. 1999;2:69-78.

16. Brundin T, Wahren J. Effects of i.v. amino acids on human splanchnic and whole body oxygen consumption, blood flow, and blood temperatures. Am J Physiol. 1994;266:E396-402.

17. Yamaoka I, Doi M, Nakayama M, Ozeki A, Mochizuki S, Sugahara K, et al, Intravenous administration of amino acids during anesthesia stimulates muscle protein synthesis and heat accumulation in the body. Am J Physiol Endocrinol Metab. 2006;290:E882-888.

18. Yamaoka I, Doi M, Kawano Y, Nakayama M, Watanabe Y, Oba K, et al. Insulin mediates the linkage acceleration of muscle protein synthesis, thermogenesis, and heat storage by amino acids. Biochem Biophys Res Commun. 2009;386:252-6.

19. Widman J, Hammarqvist F, Sellden E. Amino acid infusion induces thermogenesis and reduces blood loss during hip arthroplasty under spinal anesthesia. Anesth Analg. 2002;95:1757-62. table of contents.

20. Krebs M, Brehm A, Krssak M, Anderwald C, Bernroider E, Nowotny P, et al. Direct and indirect effects of amino acids on hepatic glucose metabolism in humans. Diabetologia. 2003:46:917-25.

21. Felig P, Marliss E, Cahill Jr GF. Are plasma amino acid levels elevated in obesity? N Engl J Med. 1970;282:166.

22. Felig P, Marliss E, Cahill Jr GF. Plasma amino acid levels and insulin secretion in obesity. N Engl J Med. 1969;281:811-6.

23. Tremblay F, Lavigne $\mathrm{C}$, Jacques $\mathrm{H}$, Marette $\mathrm{A}$. Role of dietary proteins and amino acids in the pathogenesis of insulin resistance. Annu Rev Nutr. 2007:27:293-310.

24. Newgard CB, An J, Bain JR, Muehlbauer MJ, Stevens RD, Lien LF, et al. A branched-chain amino acid-related metabolic signature that differentiates obese and lean humans and contributes to insulin resistance. Cell Metab. 2009;9:311-26.

25. Krebs M, Krssak M, Bernroider E, Anderwald C, Brehm A, Meyerspeer M, et al. Mechanism of amino acid-induced skeletal muscle insulin resistance in humans. Diabetes. 2002;51:599-605.

26. Patti ME, Brambilla E, Luzi L, Landaker EJ, Kahn CR. Bidirectional modulation of insulin action by amino acids. J Clin Invest. 1998:101:1519-29.

27. Nordenstrom J, Sonnenfeld T, Arner P. Characterization of insulin resistance after surgery. Surgery. 1989;105:28-35.

28. Brandi LS, Frediani M, Oleggini M, Mosca F, Cerri M, Boni C, et al. Insulin resistance after surgery: normalization by insulin treatment. Clin Sci (Lond). 1990;79:443-50. 